\begin{abstract}
Friendship experiences have been shown to be important predictors of adolescents' loneliness. The current study examined selection and socialization effects of loneliness within reciprocal best friendships, while controlling for friendship quality. Analyses were conducted on a sample of 884 adolescents ( $42.08 \%$ boys), making up 442 dyads, who were on average 13.51 years old $(S D=1.37)$. Adolescents completed the peer-related loneliness subscale of the Loneliness and Aloneness Scale for Children and Adolescents as well as the Friendship Qualities Scale. A longitudinal actor-partner interdependence model, which accounts for the interdependencies in the data of best friends, suggested the presence of a selection effect for loneliness but no socialization effect. This finding within best friendships contrasts with studies on friendship networks where both selection and socialization were found.
\end{abstract}

Keywords: Loneliness, best friendship, friendship quality, Actor-Partner Interdependence Model 


\section{Lonely Adolescents and Their Best Friend: An Examination of Loneliness and Friendship Quality in Best Friendship Dyads}

Loneliness is a negative feeling that arises from an experienced discrepancy between the desired and perceived quality or quantity of social relationships (Perlman \& Peplau, 1981). Although loneliness is experienced across the lifespan, it seems to peak during adolescence (e.g., Heinrich \& Gullone, 2006; Qualter et al., 2015). During the transition to adolescence, substantial changes in the social world of youngsters occur that increase the risk for developing feelings of loneliness (Laursen \& Hartl, 2013). For example, adolescents increasingly spend time with their peers, especially in unsupervised contexts, as compared to with their parents (Larson \& Richards, 1991). Establishing and maintaining qualitative friendships with peers is often seen as one of the most important developmental tasks during adolescence (Steinberg \& Morris, 2001). Not being able to fulfil these developmental tasks puts adolescents at risk for experiencing loneliness (Qualter et al., 2015).

Previous studies have shown that various peer experiences have a unique contribution to loneliness (e.g., Parker \& Asher, 1993; Vanhalst, Luyckx, \& Goossens, 2014). Of these peer experiences, dyadic friendship experiences, such as friendship quality and friendship quantity, proved to be stronger predictors of adolescent loneliness than group experiences, such as victimization and social acceptance (Vanhalst et al., 2014).

Friends tend to be more similar to one another than non-friends, a tendency called homophily, as the result of selection and socialization processes (Giletta et al., 2011; Laursen, Popp, Burk, Kerr, \& Stattin, 2008). Selection is a process in which individuals befriend peers with characteristics similar to themselves. Socialization is a process through which friends become more similar in their characteristics due to their mutual influence on each other (Laursen et al., 2008). Two studies, using social network analyses, provided evidence for both selection and socialization effects of loneliness within networks of friends (Cacioppo, Fowler, 
\& Christakis, 2009; Mercer \& Derosier, 2010). More specifically, individuals with similar loneliness levels were more likely to become friends. In addition, dyad members were found to become more similar in their loneliness levels over time (Cacioppo et al., 2009; Mercer \& Derosier, 2010). As these studies used friendship networks, it remains unclear whether similar selection and socialization effects occur at the level of best friendships. Findings regarding friendships might not be generalizable to best friendships because friendships and best friendships are substantially different, for example in their level of closeness and intimacy (see Berndt \& McCandless, 2009).

Friendship quality might influence loneliness homophily. The influence of friends on each other appears to be stronger when friendships are of higher quality (e.g., Berndt, 2002; Berndt \& Keefe, 1995; Giletta et al., 2011). Yet, loneliness is consistently related to reports of low friendship quality (e.g., Cacioppo et al., 2009; Kingery, Erdley, \& Marshall, 2011; Lodder, Scholte, Goossens, \& Verhagen, 2015; Nangle, Erdley, Newman, Mason, \& Carpenter, 2003; Vanhalst et al., 2014). In other words, it is possible that the low friendship quality associated with loneliness results in weakened loneliness homophily. On the one hand, low perceived friendship quality increases the likelihood that one's desired level of friendship quality is not met. Such unfulfilled desire could result in higher reports of loneliness (Perlman \& Peplau, 1981). On the other hand, loneliness is associated with socially inhibited behavior (e.g., Jobe-Shields, Cohen, \& Parra, 2011), which might hamper the maintenance of high quality friendships (Cacioppo \& Hawkley, 2009).

The current study aimed to examine loneliness and best friendship reciprocity as well as loneliness homophily processes in adolescents' best friendships. More specifically, we aimed to examine selection and socialization effects of loneliness within best friend dyads, while controlling for friendship quality. First, we expect that dyad members become friends with peers who are similar in their loneliness levels (i.e., selection effects). Second, we expect 
that dyad members become more similar over time (i.e., socialization effects) in their loneliness levels. Thus, loneliness as reported by a particular adolescent is expected to predict that person's best friend's report of loneliness one year later, and vice versa.

\section{Method}

\section{Participants}

The total sample consisted of 2,340 adolescents ( $46.94 \%$ boys) between 10 and 20 years old $(M=13.80, S D=1.44)$ enrolled in nine schools in the Dutch-speaking part of Belgium. Of the total sample, 1.541 adolescents $(65.86 \%)$ were present at both measurement occasions, 469 adolescents $(20.04 \%)$ were only present at T1 and 330 adolescents $(14.10 \%)$ were only present at T2 (one year later). Little's MCAR Test (Little, 1988), using expectation maximization estimation, revealed a normed $\chi^{2}$ of 1.24 , which indicated that the data were missing at random (Ulman, 2013). The majority of adolescents (92.94\%) were of Belgian origin, $4.54 \%$ of the adolescents could trace back their origin to another European country, and $2.52 \%$ of the adolescents were of non-European origin. Most adolescents were enrolled in the academic track $(85.27 \%), 11.42 \%$ were enrolled in the technical track, and $3.31 \%$ were enrolled in the vocational track. A total of 280 adolescents $(14.07 \%)$ were enrolled in Grade 6, which corresponds to the last year in elementary school, 740 in Grade 7 (37.19\%), which is the first year of secondary school, $353(17.74 \%)$ in Grade 8 (i.e., second year of secondary school), 321(16.13\%) in Grade 9 (i.e., third year of secondary school), 295 (14.87\%) in Grade 10 (i.e., fourth year of secondary school), and 400 adolescents did not indicate their grade at any time point.

\section{Procedure}

Participants were drawn from the second and third wave of data collection (from now on abbreviated as T1 and T2, respectively) of a larger longitudinal project [reference omitted for blinded review]. The first wave of data collection was not used, as the best friendship 
nomination procedure was only included from the second wave on. There was approximately one year between the measurement occasions. Before the start of the study, prospective participants and their parents received an information letter, through the school, about the study. Passive consent was obtained from parents, who were asked to sign the information letter and hand it back to the teacher if they did not want their child participating in the study. Active, written consent was obtained from adolescents at the assessment day, after they were informed that participation was voluntary and confidential. The questionnaires were completed in pencil-and-paper format during regular school hours for two subsequent years in the presence of a research assistant. In return for participation, participants received a small gift (e.g., sweets) and cinema tickets were raffled. Ethical approval was obtained from the Internal Review Board of the university (S55360).

Reciprocal best friendships were determined by examining whether the nominated best friend reciprocated the nomination. Probably due to the use of an unlimited nomination method, half of the sample $(n=1,396,59.66 \%)$ did not have their best friendship nomination reciprocated at any time point. As these adolescents were never involved in a reciprocal friendship, they were not included in the APIM analyses. In addition, 70 adolescents $(2.99 \%)$ were involved in different reciprocal best friendships across the two measurement occasions. Adolescents involved in such instable reciprocal best friendships would contribute disproportionally to the results if they were included in the APIM analyses. Therefore, it has been suggested to randomly select a dyad at either time point to restrict each adolescent to one friendship dyad (e.g., Giletta et al., 2011; Popp, Laursen, Kerr, Stattin, \& Burk, 2008). As a result, an additional 60 adolescents had to be excluded from the APIM analyses as they lost their reciprocal friendship, which resulted in a total of 1,456 adolescents $(62.22 \%)$ without any reciprocal best friendship. A total of 884 adolescents $(37.78 \%$ of total sample; $42.08 \%$ boys), who were on average 13.51 years old $(S D=1.37)$, had a reciprocal best friend on at 
least one measurement occasion, making up 442 dyads. Of these adolescents with a reciprocal friendship on at least one measurement occasion, 392 (16.75\%) adolescents had dissolving best friendships, that is, they had a reciprocal friendship at T1 only. Furthermore, 304 adolescents (12.99\%) were involved in nascent best friendships, that is, they were involved in a reciprocal friendships at T2 only. Finally, 188 adolescents $(8.03 \%)$ had stable reciprocal best friends. Attrition analyses indicated that adolescents without any reciprocal friendship were significantly older and reported more loneliness and less friendship quality than adolescents with a reciprocal friendship (see Table 1).The finding that those without a reciprocal friendship were lonelier might be explained by the finding that loneliness at $\mathrm{T} 1$ was related to a decreased likelihood of nominating a classmate $(O R=0.70,95 \% \mathrm{CI}[0.58,0.84])$, or a peer from one's grade $(O R=0.74,95 \% \mathrm{CI}[0.59,0.92])$ in comparison to nominating a peer not enrolled at one's current school. At T2, loneliness was also related to a decreased likelihood of nominating a classmate $(O R=0.67,95 \% \mathrm{CI}[0.53,0.81])$ over nominating a peer not enrolled at one's school, but not to nominating a peer from one's grade $(O R=0.92$, $95 \%$ CI $[0.73,1.16])$ or school $(O R=0.75,95 \% \mathrm{CI}[0.47,1.19])$. Being more likely to nominate a peer not enrolled at one's school as best friends subsequently decreases the likelihood of having a reciprocal best friendship (as the peer is unlikely to participate in the current study).

\section{Measures}

Friendship quality. Friendship quality was assessed with the Friendship Qualities Scale (FQS; Bukowski, Hoza, \& Boivin, 1994). This scale is composed of 23 items that tap both positive and negative aspects of a friendship, that is, the experienced companionship, conflict, help, security, and closeness within a relationship. Sample items are "My friend and I go to each other's houses after school and on weekends", "I can get into fights with my friend" and "My friend would help me if I needed it". While thinking of the person they 
nominated as their best friend, participants rated the statements about their friendship on a 5point Likert scale ranging from 1 (not at all true) to 5 (really true). A mean friendship quality score was computed, for which the items of the conflict scale were reversed. A higher score indicated better friendship quality. The questionnaire was reliable, Cronbach's $\alpha=.91$ at both measurement occasions.

Loneliness. Peer-related loneliness was assessed using the peer-related loneliness subscale of the Loneliness and Aloneness Scale for Children and Adolescents (Goossens, 2016). The scale consists of 12 items (e.g., "I think I have fewer friends than others"; "I feel excluded by my classmates"; "I feel alone at school"), which were rated on a 4-point Likert scale, ranging from 1 (never) to 4 (often). A higher score indicated more peer-related loneliness. The questionnaire was reliable, Cronbach's $\alpha=.91$ at both measurement occasions).

Best friendship nomination. We further asked for the name of respondents' best friend. This was an open question, with no requirement that the friend would be in the same school.

\section{Data Analysis}

The degree of member indistinguishability was tested with intraclass correlations (ICC; Kenny, Kashy, \& Cook, 2006). For loneliness at T1, ICC estimates ranged between .11 and .28 for the various friendship groups. At T2 the ICC estimated for loneliness ranged between .02 and .20 for the various friendship group. For friendship quality, ICC estimated ranged between .20 and .52 at $\mathrm{T} 1$ and between .15 and .53 at $\mathrm{T} 2$ for the various friendship groups. In other words, the ICCs show a small to moderate degree of similarity, respectively for loneliness and friendship quality, between dyad members.

Several longitudinal actor-partner interdependence model (APIM; Kenny et al., 2006) were estimated in Mplus Version 7.31 (Muthén \& Muthén, 1998-2012) to account for the 
interdependence between best friend dyad members and to reduce biased significant tests (Laursen et al., 2008). The fit indices were adjusted for dyad indistinguishability (Peugh, DiLillo, \& Panuzio, 2013). Three different models were estimated as recommended by Kenny et al. (2006). First, a null model was estimated, in which all path effects were fixed to zero. Second, a saturated model was estimated. Such model includes all possible effects, but constrains similar paths between dyad members to be equal. Constraining the paths between dyad members to be equal is needed to control for the interchangeable nature of the dyad members, and allows the saturated model to have some degrees of freedom (Kenny et al., 2006). Figure 1 displays the fully saturated longitudinal APIM measurement model. In this model, paths A, B, C, D, M, and S represent actor effects, that is, the effects of the individuals' predictors on their own outcomes. On the other hand, the paths E, F, G, H, N, T, $\mathrm{U}, \mathrm{V}, \mathrm{W}$, and $\mathrm{X}$ represent partner effects, that is, the effect of the individuals' predictors on their best friends' outcomes. Third, the model of interest was estimated. This model is derived from the saturated model by systematically trimming the non-significant effects by (a) estimating the model without non-significant within-person effects; (b) estimating the model without non-significant between-person effects; and (c) estimating the model without nonsignificant within- and between-person effects (Kenny et al., 2006). Fit indices of this model of interest were then corrected by using the null and saturated model (Peugh et al., 2013).

We used a multi-group approach to disentangle selection and socialization effects (e.g., Popp et al., 2008). An initial dyadic similarity or selection effect is indicated by a significant concurrent correlation between loneliness or friendship quality reports of the dyad members (i.e., paths $\mathrm{U}$ and V), while controlling for possible socialization effects. Socialization effects can be found in a significant residual correlation between loneliness or friendship quality reports of the dyad members in enduring friendship (i.e., paths $\mathrm{W}$ and $\mathrm{X}$ ), while controlling for selection effects (Giletta et al., 2011; Popp et al., 2008). The effects of 
one friend on the other (i.e., cross-paths E, F, G, and H) should be considered as error in analyses with indistinguishable dyads (Laursen et al., 2008).

\section{Results}

Table 2 summarizes the correlations, means, and standard deviations for the study variables. Using the model as depicted in Figure 1, we tested whether the three friendship groups (i.e., dissolving, nascent, and stable friendship groups) could be constrained to a single model. The model in which the friendship groups were constrained to be equal did not fit the data any worse than the unconstrained model $\left(\Delta \chi^{2}(30)=30.65, p=.433\right)$. This suggested that there were no significant differences between dissolved, nascent, and stable friendships. As the constrained model was more parsimonious, it was used for further analyses. We then removed the non-significant effects from the model to derive the model of interest. Although removing the non-significant actor effects did not affect the model fit $\left(\Delta \chi^{2}(2)=4.84, p=\right.$ .089), removing the non-significant partner effects did decrease the model fit $\left(\Delta \chi^{2}(7)=55.04\right.$, $p<.001)$. Therefore, the final model was the model without non-significant actor effects, but with the non-significant partner effects (see Figure 2). This model fitted the data well $\left(\chi^{2}(4)=\right.$ $5.14, p=.273, \mathrm{RMSEA}=.03, \mathrm{CFI}=1, \mathrm{TLI}=.99)$.

At T1, self-reported loneliness was negatively correlated with self-reported friendship quality (i.e., path $\mathrm{M}$ in Figure 1). In other words, higher loneliness levels were related to lower friendship quality. Moreover, both reports of loneliness and friendship quality were correlated between the dyad members (i.e., path $\mathrm{U}$ in Figure 1). That is, higher loneliness as reported by one dyad member was related to higher loneliness as reported by the best friend. Similarly, higher friendship quality as reported by one dyad member was related to higher friendship quality as reported by the best friend (i.e., path V in Figure 1). Loneliness as reported by one dyad member was related to lower friendship quality as reported by the best 
friend (i.e., path $\mathrm{N}$ in Figure 1). However, it should be noted that this correlation only became significant after removing the non-significant actor effects $\left(p_{\text {saturated }}\right.$ model $\left.=.051\right)$.

Self-reported loneliness predicted one's own loneliness one year later. Similarly, selfreported friendship quality predicted one's own friendship quality one year later. Moreover, a relative increase in loneliness over time was associated with a correlated decrease in selfreported friendship quality (i.e., actor correlated change; path S in Figure 1). However, selfreported loneliness did not predict one's own reports of friendship quality one year later, or vice versa (i.e., actor cross-paths; paths $\mathrm{C}$ and $\mathrm{D}$ in Figure 1). Neither did loneliness or friendship quality of one dyad member predict levels of loneliness or friendship quality of his or her best friend (i.e., partner cross-paths; paths E, F, G, and G in Figure 1).

\section{Discussion}

Previous studies indicated that loneliness is related to being less likely to have a reciprocal best friend (e.g., Lodder et al., 2015; Nangle et al., 2003). However, it remained unclear whether the selection and socialization effects of loneliness between friends (Cacioppo et al., 2009; Mercer \& Derosier, 2010) were similar to the selection and socialization effects of loneliness between best friends. Therefore, in the current study, we aimed to examine loneliness homophily processes, while controlling for friendship quality, within best friend dyads. The results of our study indicated that initial loneliness was related to initial loneliness of the best friend and lower initial best friendship quality, as reported by both friends, indicating a selection effect. A socialization effect was found for friendship quality, but not for loneliness.

Best friends appeared to be more similar in their initial loneliness levels, suggesting the presence of a selection effect. Because we were unable to distinguish between stable, dissolving, and nascent friendships, we want to emphasize that this finding should be interpreted with caution. That is, the inability to distinguish between stable, dissolving, and 
nascent friendships suggests that similarity in loneliness at $\mathrm{T} 1$ is related to becoming friends, dissolving a friendship, or maintaining the friendship. A speculative interpretation of this finding is that lonely individuals are in desperate need of a friend and, therefore, start a friendship with any available peer. Although they might remain friends with such a peer, because no one else is available, it is not a good basis to start a friendship.

In contrast to suggesting the presence of a selection effect, our study did not indicate that loneliness levels of best friends became more similar over time. Thereby, our study suggest that the socialization effects found in friendship networks (Cacioppo et al., 2009; Mercer \& Derosier, 2010) are not necessarily generalizable to socialization effects within dyadic friendships. However, a limitation of our study is that we showed that lonely individuals were more likely to nominate someone outside of school as their best friend, who often did not participate in our study, and were subsequently less likely to have a reciprocal best friend. As a result, those without reciprocal best friendships, who were not included in the APIM analyses, were found to be lonelier than those with a reciprocal friendship. Thereby, our findings based on the APIM analyses for reciprocal dyads are inherently not generalizable to the more lonely individuals. Replication of our findings with a more representative sample is needed. However, it should be noted that there was sufficient variation in loneliness in the current study. It is possible that this limitation might explain the difference in homophily effects between the current study and the two network studies, because the latter adopted a more population-based approach (Cacioppo et al., 2009; Mercer \& Derosier, 2010).

The current study indicated that initial loneliness was related to initial lower self- and best-friend-reported friendship quality. These findings seem to be in line with the results of a previous, cross-sectional study (Lodder et al., 2015). The association between loneliness and lower self-reported friendship quality might be an indication of a cognitive bias. The 
cognitive bias hypothesis suggests that loneliness is related to a more negative or less positive evaluation of one's relationships with others (Cacioppo \& Hawkley, 2009; Qualter et al., 2015). Besides reflecting a cognitive bias, it is also possible that the link between greater loneliness and lower self-reported friendship quality might reflect involvement in friendships that are actually of lower quality. That is, loneliness has been associated with negative characteristics, such as being less communicatively competent (Tsai \& Reis, 2009), inappropriate self-disclosure (Burke, Woszidlo, \& Segrin, 2012; Tsai \& Reis, 2009; Wei, Russell, \& Zakalik, 2005) and general social skill deficits (e.g., Lodder, Goossens, Scholte, Engels, \& Verhagen, 2016; Schinka, van Dulmen, Mata, Bossarte, \& Swahn, 2013) that might elicit lower quality friendships. The association between one's initial loneliness with lower reported friendship quality by the best friend would be in line with this type of interpretation. Yet, contradicting such reasoning was the finding that an increase of loneliness over time was not related to a decrease in friendship quality as reported by the best friend, or vice versa.

In sum, the current findings suggest that selection and socialization effects within best friendships might differ from the selection and socialization effect previously found in friendship networks. That is, the current study found support for selection, but not socialization of loneliness within best friendship dyads.

\section{References}

Berndt, T. J. (2002). Friendship quality and social development. Current Directions in Psychological Science, 11, 7-10. doi:10.1111/1467-8721.00157

Berndt, T. J., \& Keefe, K. (1995). Friends' influence on adolescents' adjustment to school. Child Development, 66, 1312-1329. doi:10.2307/1131649

Berndt, T. J., \& McCandless, M. A. (2009). Methods for investigating children's relationships with friends. In K. H. Rubin, W. M. Bukowski, \& B. Laursen (Eds.), Handbook of peer interactions, relationships and groups (pp. 63-81). London, UK: Guilford Press. 
Bukowski, W. M., Hoza, B., \& Boivin, M. (1994). Measuring friendship quality during preand early adolescence: The development and psychometric properties of the Friendship Qualities Scale. Journal of Social and Personal Relationships, 11, 471484. doi:10.1177/0265407594113011

Burke, T. J., Woszidlo, A., \& Segrin, C. (2012). Social skills, family conflict, and loneliness in families. Communication Reports, 25, 75-87. doi:10.1080/08934215.2012.719461

Cacioppo, J. T., Fowler, J. H., \& Christakis, N. A. (2009). Alone in the crowd: The structure and spread of loneliness in a large social network. Journal of Personality \& Social Psychology, 97, 977-991. doi:10.1037/a0016076

Cacioppo, J. T., \& Hawkley, L. C. (2009). Perceived social isolation and cognition. Trends in cognitive sciences, 13, 447-454. doi:10.1016/j.tics.2009.06.005

Giletta, M., Scholte, R. H. J., Burk, W. J., Engels, R. C. M. E., Larsen, J. K., Prinstein, M. J., \& Ciairano, S. (2011). Similarity in depressive symptoms in adolescents' friendship dyads: Selection or socialization? Developmental Psychology, 47, 1804-1814. doi:10.1037/a0023872

Goossens, L. (2016). Louvain Loneliness and Aloneness scale for Children and Adolescents [Leuvense Eenzaamheidsschaal voor kinderen en adolescenten]. Leuven, BE: Acco.

Heinrich, L. M., \& Gullone, E. (2006). The clinical significance of loneliness: A literature review. Clinical Psychology Review, 26, 695-718. doi:10.1016/j.cpr.2006.04.002

Jobe-Shields, L., Cohen, R., \& Parra, G. R. (2011). Patterns of change in children's loneliness: Trajectories from third through fifth grades. Merrill-Palmer Quarterly, 57, 25-47. doi:10.1353/mpq.2011.0003

Kenny, D. A., Kashy, D. A., \& Cook, W. (2006). Dyadic data analysis. New York, NY: Guilford Press. 
Kingery, J. N., Erdley, C. A., \& Marshall, K. C. (2011). Peer acceptance and friendship as predictors of early adolescents' adjustment across the middle school transition. Merrill-Palmer Quarterly, 57, 215-243. doi:10.1353/mpq.2011.0012

Larson, R. W., \& Richards, M. H. (1991). Daily companionship in late childhood and early adolescence: Changing developmental contexts. Child Development, 62, 284-300. doi:10.2307/1131003

Laursen, B., \& Hartl, A. C. (2013). Understanding loneliness during adolescence: Developmental changes that increase the risk of perceived social isolation. Journal of Adolescence, 36, 1261-1268. doi:10.1016/j.adolescence.2013.06.003

Laursen, B., Popp, D., Burk, W., Kerr, M., \& Stattin, H. (2008). Incorporating interdependence into developmental research: Examples from the study of homophily and homogeneity. In N. A. Card, J. P. Selig, \& T. D. Little (Eds.), Modeling dyadic and interdependent data in developmental research (pp. 11-38). Mahwah, NJ: Erlbaum.

Little, R. J. (1988). A test of missing completely at random for multivariate data with missing values. Journal of the American Statistical Association, 83, 1198-1202. doi:10.2307/2290157

Lodder, G. M. A., Goossens, L., Scholte, R. H. J., Engels, R. C. M. E., \& Verhagen, M. (2016). Adolescent loneliness and social skills: Agreement and discrepancies between self-, meta-, and peer-evaluations. Journal of Youth and Adolescence, 44, 1441-1456. doi:10.1007/s 10964-016-0461-y

Lodder, G. M. A., Scholte, R. H., Goossens, L., \& Verhagen, M. (2015). Loneliness in early adolescence: Friendship quantity, friendship quality, and dyadic processes. Journal of Clinical Child \& Adolescent Psychology, 1-12. doi:10.1080/15374416.2015.1070352 
Mercer, S. H., \& Derosier, M. E. (2010). Selection and socialization of internalizing problems in middle childhood. Journal of Social and Clinical Psychology, 29, 1031-1056. doi:10.1521/jscp.2010.29.9.1031

Muthén, L. K., \& Muthén, B. O. (1998-2012). Mplus user's guide (7th ed.). Los Angeles, CA: Muthén \& Muthén.

Nangle, D. W., Erdley, C. A., Newman, J. E., Mason, C. A., \& Carpenter, E. M. (2003). Popularity, friendship quantity, and friendship quality: Interactive influences on children's loneliness and depression. Journal of Clinical Child and Adolescent Psychology, 32, 546-555. doi:10.1207/S15374424JCCP3204_7

Parker, J. G., \& Asher, S. R. (1993). Friendship and friendship quality in middle childhood: Links with peer group acceptance and feelings of loneliness and social dissatisfaction. Developmental Psychology, 29, 611-621. doi:10.1037/0012-1649.29.4.611

Perlman, D., \& Peplau, L. A. (1981). Toward a social psychology of loneliness. In R. Gillmour \& S. Duck (Eds.), Personal relationships Vol. 3: Personal relationships in disorders (pp. 31-56). London, UK: Academic Press.

Peugh, J. L., DiLillo, D., \& Panuzio, J. (2013). Analyzing mixed-dyadic data using Structural Equation Models. Structural Equation Modeling: A Multidisciplinary Journal, 20, 314-337. doi:10.1080/10705511.2013.769395

Popp, D., Laursen, B., Kerr, M., Stattin, H., \& Burk, W. J. (2008). Modeling homophily over time with an Actor-Partner Interdependence Model. Developmental Psychology, 44, 1028-1039. doi:10.1037/0012-1649.44.4.1028

Qualter, P., Vanhalst, J., Harris, R., Van Roekel, E., Lodder, G., Bangee, M., . . . Verhagen, M. (2015). Loneliness across the life span. Perspectives on Psychological Science, 10, 250-264. doi:10.1177/1745691615568999 
Schinka, K. C., van Dulmen, M. H., Mata, A. D., Bossarte, R., \& Swahn, M. (2013). Psychosocial predictors and outcomes of loneliness trajectories from childhood to early adolescence. Journal of Adolescence, 36, 1251-1260. doi:10.1016/j.adolescence.2013.08.002

Steinberg, L., \& Morris, A. S. (2001). Adolescent development. Annual Review of Psychology, 52, 83-110. doi:10.1146/annurev.psych.52.1.83

Tsai, F. F., \& Reis, H. T. (2009). Perceptions by and of lonely people in social networks. Personal Relationships, 16, 221-238. doi:10.1111/j.1475-6811.2009.01220.x

Ulman, J. B. (2013). Structural equation modeling. In B. G. Tabachnick \& L. S. Fidell (Eds.), Using multivariate statistics (6th ed., pp. 681-785). Boston, MA: Pearson Education.

Vanhalst, J., Luyckx, K., \& Goossens, L. (2014). Experiencing loneliness in adolescence: A matter of individual characteristics, negative peer experiences, or both? Social Development, 23, 100-118. doi:10.1111/sode.12019

Wei, M., Russell, D. W., \& Zakalik, R. A. (2005). Adult attachment, social self-efficacy, selfdisclosure, loneliness, and subsequent depression for freshman college students: A longitudinal study. Journal of Counseling Psychology, 52, 602. doi:10.1037/00220167.52.4.602 


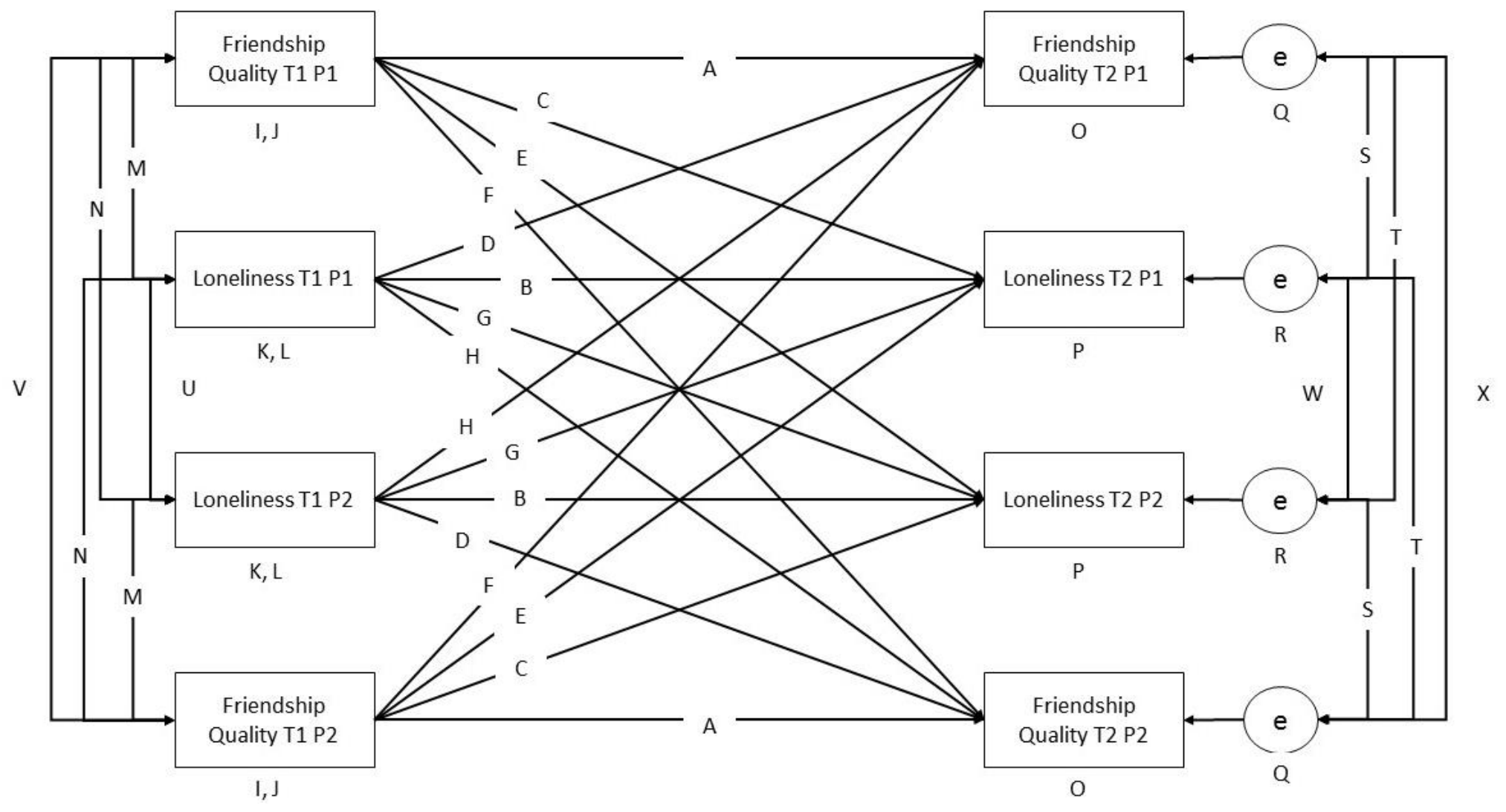

Figure 1. Measurement model for indistinguishable dyad assessing longitudinal associations between loneliness and friendship quality.

Note: the model includes stability paths $(A$ and $B)$, within-person paths $(C$ and $D)$, between-person paths $(E, F$, $G$ and $H)$, within-person correlations $(M$ and $S)$ and between-person correlations $(N, T, U, V, W, X)$. Letters $\mathrm{I}, \mathrm{K}, \mathrm{O}$ and $\mathrm{P}$ indicate intercepts/mean, $\mathrm{J}$ and $\mathrm{L}$ indicate 
variance, $\mathrm{R}$ and $\mathrm{Q}$ indicate residual variance.

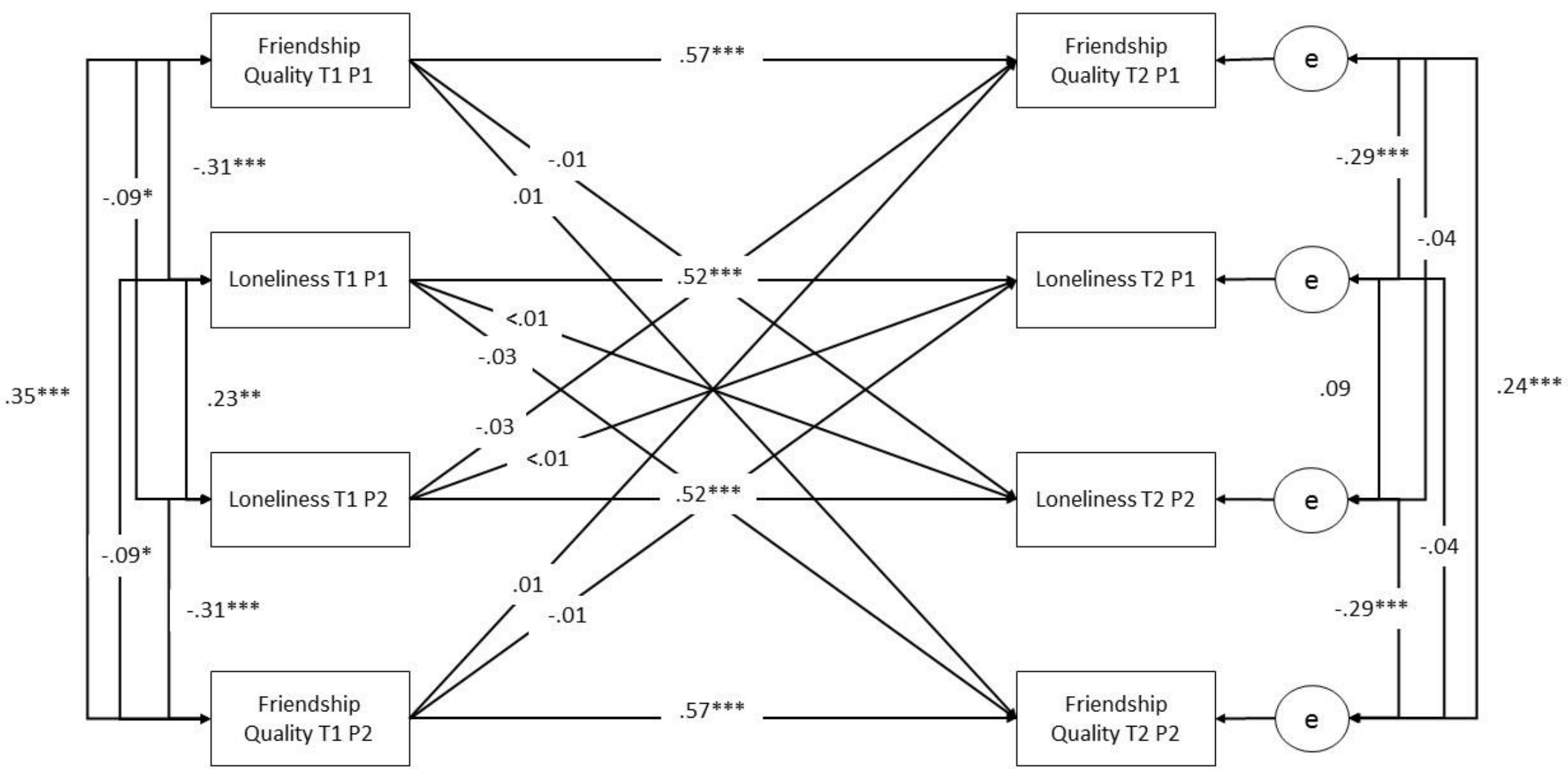

Figure 2. Observed longitudinal associations between loneliness and friendship quality within best friendship dyads. 
Table 1

Comparison between Adolescents Without any Reciprocal Friend and Adolescents with a Reciprocal Friend on age, Loneliness and Friendship Quality

\begin{tabular}{lccccc}
\hline \multicolumn{1}{c}{ Variable } & $\begin{array}{c}M \text { Without } \\
\text { reciprocal friend }\end{array}$ & $\begin{array}{c}M \text { With a } \\
\text { reciprocal friend }\end{array}$ & $t$ & $d f$ & $p$ \\
\hline Age & 13.90 & 13.66 & 3.96 & 1969.34 & .008 \\
Loneliness T1 & 1.72 & 1.56 & 6.08 & 1901.75 & $<.001$ \\
Loneliness T2 & 1.64 & 1.57 & 2.82 & 1673.34 & .005 \\
Friendship quality T1 & 3.70 & 3.87 & -6.32 & 1743.59 & $<.001$ \\
Friendship quality T2 & 3.77 & 3.86 & -3.35 & 1832 & .001 \\
\hline
\end{tabular}


Table 2

Correlations, Means, and Standard Deviations of Loneliness and Friendship Quality

\begin{tabular}{lcccccc}
\hline Variable & 1. & 2. & 3. & 4. & $M$ & $S D$ \\
\hline $\begin{array}{l}\text { 1. Peer-related } \\
\text { loneliness T1 }\end{array}$ & - & & & & 1.65 & 0.61 \\
$\begin{array}{l}\text { 2. Peer-related } \\
\text { loneliness T2 }\end{array}$ & .57 & - & & & 1.61 & 0.57 \\
$\begin{array}{l}\text { 3. Friendship } \\
\text { quality T1 }\end{array}$ & -.33 & -.23 & - & & 3.77 & 0.58 \\
$\begin{array}{l}\text { 4. Friendship } \\
\text { quality T2 }\end{array}$ & -.23 & -.36 & .57 & - & 3.81 & 0.55 \\
\hline
\end{tabular}

Note: $\mathrm{T} 1=$ the first measurement occasion; $\mathrm{T} 2=$ the second measurement occasion. All correlations were $p<.001$. 